\title{
Recent advances in digital particle image velocimetry methods for flow motion analysis
}

\author{
H. Nasibov ${ }^{1, \star}$ and S. Baytaroglu ${ }^{2, \star \star}$ \\ 1 National Research Institute of Electronics \&Cryptology, TUBITAK-UEKAE, Gebze, Kocaeli, Turkey \\ 2 National Metrology Institute, TUBITAK-UME, Gebze, Kocaeli, Turkey
}

Received: 15 December 2009 / Accepted: 5 February 2010

\begin{abstract}
The advent of charge-coupled devices (CCD) has significantly extended the horizons of full-field measurement methods in many fields of fundamental and applied sciences, including fluid flow dynamics. One of the major applications of imaging sensors in fluid mechanics is the Digital Particle Image Velocimetry (DPIV), which is a non-invasive, full field optical measuring technique. Due to recent advances in image processing methods, and in digital imaging, laser and optics DPIV has become a dominant tool for obtaining velocity information about fluid motion. On the other hand, over the last years, developments in micro- and nanofluidic systems have promised a shrinking of the desktop-sized chemical and biological devices to microscale size, so a detailed investigation of the behaviour of flow inside these microdevices is essential for the optimum design of microsystems, as well as for an understanding of flow dynamics in micron scales. In this work, the advances in visualization and quantitative measurements of flow velocity measurements are reviewed and explained.
\end{abstract}

Keywords: $\mu \mathrm{PIV}$; microflow; cross-correlation; PIV; PTV; seeding particles

\section{Introduction}

2009 Nobel Prize in Physics was partly awarded to Boyle and Smith "for the invention of an imaging semiconductor circuit - the CCD sensor" [1]. Over the last 40 years the achievements in semiconductor imaging sensors have been recognized by the scientific community as "tested by time". Digital cameras, based on these imaging sensors have widely penetrated into many fields of science and industry, from astronomy to microscopy, from process control to the measurements of moving objects, etc. Scientific grade digital cameras, due to their high sensitivity, linearity, high signal-to noise ratio, and perfect photometric parameters have been recognized as highly stable sensors and are now used in many areas of metrology.

One of the major applications of optical imaging sensors is in diagnostics and investigations of fluid flow dynamics. In recent years, the progress and achievements in fluid and gas flow visualization methods as well digital image sensor technology have made it possible to analyze and measure the flow velocities in many applications with high accuracy.

Historically, the first flow velocity measurement instrument (Pitot-static tube) was invented by a French scientist, Pitot, in the early 1700's. Later, with the introduction of hot-wire anemometers in the 1920's, the first great stride in the assessment of multiple velocity components using miniature sensors was achieved. Before the

\footnotetext{
${ }^{\star}$ Correspondence: humbat@uekae.tubitak.gov.tr

${ }^{\star \star}$ Correspondence: Sakir.baytaroglu@ume.tubitak.gov.tr
}

invention of lasers, all the techniques for flow investigation were invasive in nature and required insertion of a physical probe into a flow, which in turn, intruded in the flow itself. In the early 1960's, with the invention of lasers the first non-invasive flow diagnosis methods were worked out. For example, the Laser-Doppler Anemometer (LDA) was the first non-invasive flow velocity measurement probe. In the following years, a variety of direct and indirect flow motion measurements in the macro and micro scales were worked out. Most of these systems were able to obtain velocity information at a particular point of the flow, i.e. had point-wise character. In [2] the method for investigation of electrokinetic flows by recording the current change upon replacement of one solution with a similar one of higher conductivity is described. A comprehensive review on electrokinetic flow investigation methods and techniques can be found in [3].

The next significant step in flow motion investigation was introduced with the development of visualization and recording methods. Perhaps the earliest flow visualization experiment is Reynolds' (1883), who studied the transition to turbulence in pressure-driven pipe flow. The main goal of the flow visualization is to alter the fluid in a way that the flow dynamics can be revealed, without perturbing original flow. A variety of visualization methods have been applied so far, however scalar-based, gaseous and particle-based flow visualization methods became dominant. Scalar-based visualization methods have been widely used since the time of Reynolds up to now. In scalarbased flow velocimetry, the motion of the bulk fluid is 
inferred from the observed velocity of a conserved scalar. A range of scalar visualization methods, e.g., fluorescence, photobleaching, photochromic reaction, phosphorescence, caged fluorescence, etc. are widely used in many areas of fluid physic research and fluidic device developments. An exhaustive review of visualization methods can be found in [4]. In this work, we will consider only flow measurement techniques based on the particle-based flow visualization methods.

\section{Particle-based image visualization}

In particle-based visualization methods the fluid under investigation is seeded with tracer particles. Two main characteristics of these particles are important for flow visualization: gravitational and optical. The requirements for the gravitational properties of particles arise from the fact that the particles should be able to tag the flow streamlines without excessive slip. The settling velocity $u$ of the particle under gravity, assuming that the process is governed by Stokes drag, is given by [5]:

$$
u=\frac{g d_{p}^{2}\left(\rho_{p}-\rho_{f}\right)}{18 \mu}
$$

where, $d_{p}$ is particle diameter, $\rho_{p}$ and $\rho_{f}$ are particle and fluid density, respectively, and $\mu$ is fluid viscosity. The requirement is for $u$ to be as small as possible in comparison to the actual flow velocity. As can be seen from equation (1) particles from materials with densities close to the fluid density are suitable for flow visualization. For example, polystyrene particles with density $\rho_{p}=1.05 \mathrm{~g} / \mathrm{cm}^{3}$ are good candidates for water flow velocimetry. Further steps towards diminishing the value of settling velocity can be carried out by reducing the particles diameter.

The requirements for the optical properties of tracer particles depend on the optical measurement technique employed. For scattering based techniques, particles with high reflectivity are interesting. As the reflective index of particles must be higher than the fluid, particles are coated with highly reflective materials. For example, hollow glass spheres coated with silver are a suitable material for flow visualization, and for use in experiments with water (due to its gravitational properties). More information on tracer particles can be found in [6].

One of the popular flow measurement techniques based on the particle based visualization method is the LDA (also referred to as Laser Doppler Velocimetry), which is used for the measurement of instantaneous flow velocity at a single point within a small volume. For the visualization of fluid flow, the fluid under investigation is seeded with highly reflective particles. In the small volume inside the flow, two laser beams create interference fringes and when particles cross these interference patterns, the reflection captured from the particles is recorded. Then, the flow velocity is determined from the distance between fringes and delay time. The LDA was applied for the investigation of electrokinetic flow in a closed $2 \mathrm{~mm}$ diameter capillary [7], 3D turbulent flow [8], in pressure driven flow inside a $175 \mu \mathrm{m}$ microchannel [9].

The other widely used type of tracer particle is the fluorescent polystyrene micro spheres. The polystyrene spheres are coated with fluorescent dyes. When these fluorescent dye molecules are illuminated with the electromagnetic radiation of a certain wavelength (excitation wavelength), they immediately emit a photon with a wavelength that is different from the wavelength of incident light. When the emitted photon's wavelength is larger than the wavelength of incident light, the effect is called Stoke shift. Generally, in Particle Image Velocimetry (PIV) two types of fluorescent particles, the first with an absorbing peak at $470 \mathrm{~nm}$ and emitting with a peak at about $510 \mathrm{~nm}$, and the second type, absorbing at about $535 \mathrm{~nm}$ and emitting at about $570 \mathrm{~nm}$ (Stoke shift of more than $40 \mathrm{~nm}$ ) are used. The refractive index of these particles in interested wavelength is about 1.58. The diameters of the spherical polystyrene particles vary from hundreds of nanometers up to tens of micromemters. The density of polystyrene micro spheres is very close to the density of water, so it is a suitable material for fluid experiments with distilled water.

One of the major logical extensions of flow visualization with fluorescent polystyrene micro spheres is PIV, which was introduced about 25 years ago [10]. PIV is a non-invasive, full field optical measuring technique, utilizing the particle-based visualization method. Due to the high accuracy of results and wide area of application from laminar to turbulent flow in different geometrical complexes, this method has become the dominant tool for obtaining velocity information in fluid motion [11-15].

\section{Particle image velocimetry}

In PIV experiments, the fluid of interest is seeded with fluorescent dyed free-floating probe particles. PIV requires an accurate quantitative measurement of fluid velocity vectors in a very large number of flow regions simultaneously. For this reason, a part of the flow is illuminated using a sheet of bright light, where the light sheet is parallel to the imaging plane, and its size is larger than the field of view of the imaging system. The height of the light sheet defines the depth of field of the PIV system. Thus, in contrast to the size, the light sheet's thickness must be less than the imaging system's depth of focus so that only particles that are in focus are illuminated. In order to form a light sheet of required thickness, a cylindrical lens is placed between the light source and the flow of interest. Generally, high power lasers with a wavelength corresponding to the fluorescent excitation of the tracer particles are utilized to form the light sheet. In most PIV experimental setups, illumination is performed using dual cavity pulse lasers, whose outputs reach several mJ at pulse lengths in the range of tens of nano-seconds. Other types of illuminations were also investigated, for example, in [16] the possibility and versatility of using light emitting diodes (LED) for illumination in $\mu \mathrm{PIV}$ setups was investigated. In [17] a cost-effective and miniature PIV system 


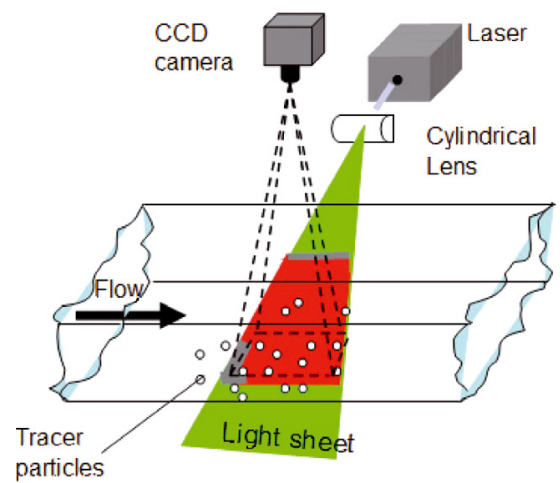

Fig. 1. Block-diagram of the PIV experimental setup.

with LED illumination, which can be used as an industrial velocity sensor, is reported.

The full field-of-view (FOV) of epifluorescent imaging optics is used to observe the illuminated area. A pass-band glass filter, with a cut-off wavelength larger than the laser wavelength and smaller than the fluorescent excitation of particles is placed in front of the image sensor to eliminate the laser illumination so that only the particle excitation light is registered. Figure 1 shows a graphical representation of the traditional PIV system.

In PIV, the position of a certain group of particles at a minimum of two instances of time is recorded by employing an image recording system. Then, from the overall displacement of particles between two consecutive image frames, the flow velocity can be estimated by [13]:

$$
\Delta X_{p} \cong V_{p} M_{0} \Delta t
$$

where, $\Delta X_{p}$ is the two-dimensional position vector in the image plane, $M_{0}$ is the optical magnification of particles in the image plane, $\Delta t$ is the time between two frames, and $V_{p}$ is the velocity vector of the particle or a particle set.

In order to calculate the displacement vector of tracer particles, corresponding frames are divided into interrogation windows, where within each interrogation window overall particle displacement vectors are calculated using cross-correlation based methods [18-20].

\section{Digital particle imaging velocimetry}

The goal of PIV experiments is to record the position of a certain group of tracer particles with high accuracy at two instances of time, while the particles are within the imaging system's field of view. There are two common methods of displacement recording in PIV experiments:

\subsection{Single frame/multi-exposure}

That method was widely used in early PIV measurements, when tracer particles images were recorded by wet-film photography. Due to the long winding time of the film between exposures, single frame/multi-exposure was the most commonly preferred recording method. Two or more exposures of the flow were made on a single frame and the tracer particles' displacement was estimated by employing the autocorrelation method. The overall process to prepare and analyze the negatives, print and digitize images, and perform displacements estimation required several hours of hard work. Taking into account that a single PIV experiment may involve hundreds of images, the complete process was very time consuming.

\subsection{Multi-frame/single-exposure}

This method is widely used in PIV based flow investigations, since it employs digital image recording sensors (CCD, CMOS, active pixel imaging sensors). Digital cameras allow one to take digitized images of flow in separate frames and to transfer the digitized signal immediately to a computer for automated analysis. The overall process of imaging, digitizing, transferring to the computer and assessment of particle displacement can be performed within a few seconds. With the employment of digital imaging equipment, PIV became one of the fastest, most accurate and dominant tools for fluid flow investigations, and came to be named DPIV (digital particle imaging velocimetry). In DPIV, two or more exposures of the particles motion, each on a separate frame, are made within a very short time span.

The digital camera plays a dominant role in DPIV systems. The two main dynamic characteristics of a DPIV system are dynamic spatial resolution and dynamic velocity resolution, which strongly depend on the performance of the particular type of camera used. The dynamic spatial range is defined by the ratio of the field of view in the object space to the smallest resolvable spatial variation [14]. This range corresponds to the number of nonoverlapping displacement vector measurements that can be done across the field of view. Higher dynamic spatial range implies better capability to assess small scale variation within the large scale fluid motion.

The main characteristics of a digital camera are determined by its electronic imaging sensor (solid state array detector). Generally, the electronic imaging sensor can be considered as a transducer of emanating light to a measurable electrical signal.

A camera sensor consists of photon-sensitive detectors, called pixels, arranged in a two dimensional area, rectangular in shape. The number of pixels in the sensor determines the spatial resolution of the CCD camera, i.e. the dynamic spatial range of the DPIV system. Nowadays, image sensors with a pixel size less than $4 \mu \mathrm{m}$ and a total number of pixels more than one million are common for conventional cameras. Besides the mechanical properties, the other characteristics of sensors, such as the ability to convert incident photons into measurable electrons (spectral sensitivity), the full well capacity (maximum number of accumulated photogenerated electrons), the transferring of the accumulated electrons via a charge-to-voltage converter according to bit depth and readout mechanism are the other important properties of imaging sensors. 
During three main processes - charge generation, charge collection and charge transferring - various types of noises (dark current noise, shot-photon noise, readout noise, etc.) are added to the signal, which affects the quality of the output image. The signal-to-noise ratio of a digital camera is another factor influencing both the spatial and the velocity dynamic ranges of a DPIV system. The dynamic velocity range of the PIV system is defined by the ratio of the maximum measureable velocity to the minimum detectable velocity [14]. In the DPIV system, the minimum detectable velocity depends on the CCD signal-to-noise ratio and spatial resolution. The other significant contribution to the limit of the minimum detectable velocity is the Brownian motion. The Brownian motion is one of the major factors limiting the lower bound of measurable flow velocity. In [21] it was shown that the Brownian motion of seeding particles could influence DPIV measurement results in certain conditions. Generally, the errors associated with the Brownian motion can be considered twofold, namely as bias and blur errors. The bias contribution is significant when the interframe time is too small, and the blur effect increases when the frame exposure time is too high. The diffusion length 1 of spherical seeding particles at room temperature in water can be defined by the Brownian diffusion coefficient:

$$
l=\sqrt{D t}
$$

where, $D$ denotes the Brownian diffusion coefficient, and $t$ denotes the time. The Brownian diffusion coefficient is defined by Einstein-Stokes equation [21]:

$$
D=\frac{k T}{3 \pi \mu d_{p}}
$$

where, $k=1.38 \times 10^{-23} \mathrm{~J} / \mathrm{K}$ is the Boltzman constant, $T$ is the thermodynamic temperature of fluid, $d_{p}$ is the radius of the seeding particles, and $\mu$ is the dynamic viscosity. A first-order error due to Brownian motion estimated relative to the displacement in one direction (in $x$ direction) is given by [22]:

$$
\varepsilon_{B}=\frac{\sqrt{s^{2}}}{\Delta x}=\frac{1}{U} \sqrt{\frac{2 D}{d t}}
$$

where, $s^{2}$ is the mean-square of particle displacement associated with the Brownian motion, $U$ is the characteristic velocity, $d t$ is the interval between two frames. Generally, $\varepsilon_{B}<1 \%$, but for particular PIV measurements, the contribution of Brownian motion to the overall measurement errors must be assessed and taken into account.

The upper limit of velocity measurements in DPIV is bounded by the characteristics of particular systems. In continuous wave laser illuminated systems, two factors, the illumination level of excited light and the full frame rate of the camera, play key roles in defining the dynamic velocity range of the micro PIV system. In low light illumination situations, it is possible to obtain a valuable signalto-noise ratio by increasing the integration time, however, this introduces significant motion blur to the particle images in addition to possibly decreasing the camera frame rate. The frame rate of CCD cameras is mainly limited by the readout time and the time necessary to transfer the charges from the sensor to a computer. For conventional cameras, this time is about $50 \mathrm{~ms}$ or higher. The camera frame rate limits the upper bound of the measurable velocity range. In the case where, due to a slow camera frame rate, the displacement of particles between two consecutive frames is greater than half of the microscope's field of view, inconsistent flow velocity measurements will be obtained due to the "loss of particle pair correspondence".

The ratio between the maximum and minimum assessable velocity measurements in a PIV system is called the dynamic velocity range and strongly depends on the instrumental parameters. This metric defines the upper limit on the resolvable velocity variation within the flow. For instance, the system will be unable to fully ascertain flow field if the dynamic velocity range is less than the magnitude of the velocity variation within the flow. As described below, the camera frame rate is one of the key factors in defining the dynamic velocity range of the DPIV system.

Depending on the readout mechanism there are two common types of CCD devices: full frame transfer and interline transfer. Full frame devices contain two identical arrays, namely the photoactive and storage, where the later is shielded from light. The photoactive array collects a charge during exposure time, and then transfers it to the storage array. While transferring charges from photoactive array to the storage array, an electromechanical shutter blocks the photoactive array. After finishing the charge transfer, the shutter opens and the photoactive array begins to exposure a new image. In parallel, the charges of previous image are being moved from the storage array to the charge-to-voltage converter and output amplifier.

In the interline transfer CCD, a light shielded storage area (vertical lines) is located within the active area of the CCD, between columns of photoactive pixels. During exposure time photoactive pixels collect a charge, and in the end, the charge from each pixel is shifted to the adjacent shielded pixel in the storage region and then transferred to output amplifier. The interline frame transfer CCDs are preferred in DPIV systems. In these sensors the readout process is performed in two steps, the first being the parallel readout step, which takes place at the end of exposure time. The charge from every pixel is simultaneously shifted directly to corresponding wells in the storage section (charge transfer channel). In the second, serial readout step, charge from the storage section is shifted row by row to output, charge-to-voltage converter and amplifier units. Detailed information about the various advantages and disadvantages of these two CCD readout techniques and about the working principles of CCD can be found, in [23].

The frame rates of conventional cameras range from 10 to $50 \mathrm{~Hz}$. High speed cameras are capable of frame rates up to $1 \mathrm{MHz}$, i.e. 1000000 frames per second. Cameras working above this range $(1 \mathrm{MHz})$ are referred to as ultrahigh speed cameras. Recently, in [24], a camera with frame rates up to $200 \mathrm{MHz}$ has been introduced. 
Other types of high speed cameras, double-frame cameras, have been worked out in the context of PIV measurements. These cameras are capable of recording a pair of full resolution frames with interframe separation of about 50 ns. In these cameras, after capturing the first frame, the charge from photo-active pixels rapidly shifts to the shielded region inside the sensor. Then, an electronic shutter opens and the sensor begins to record the next frame, while the charges of first frame are transferred to the computer. After finishing this process, an electronic shutter is closed and the charges collected in the second frame are transferred to the computer. During the charge transfer time of the second frame, the sensor remains inactive. The time between pairs of frames is about $100 \mathrm{~ms}$. In PIV measurements, the interframe timing of the doubleframe is adjusted so that each of the frames in a pair contains one pulse illumination of a dual-cavity pulse laser.

The dynamic velocity range of DPIV systems using high speed cameras and double-frame cameras is sufficient for characterization of turbulent flows where velocity drastically varies across the field of view. Contemporary PIV systems achieve dynamic velocity ranges of several hundred orders, but this still does not suffice for certain types of flows.

\section{Micro-particle image velocimetry}

The first PIV systems were designed for flow motions of millimetric and higher scales. However, the advent of the micro- and nanofluidic systems together with the advances in micromechanics and microelectronics will most likely lead to a shrinking of the desktop (even room) size chemical and biological devices to microscale integrated analytical microfluidic devices, such as Labs-on-a- chip, total analysis systems ( $\mu$-TAS), etc. In these devices, the sizes of fluid transition channels drop down to microns [25]. DPIV measurements in microscale devices are mainly restricted by the thickness of the light sheet. The approach to illuminate only a section of the micron-size channel is impractical, due to difficulties of adjusting laser sheet in micron sizes. Thus, for flow velocity measurement in microchannels, so called volume illumination is applied and the measurement region is defined by the depth of focus of a microscope objective [26-30]. The first measurement of flow velocity inside $120 \mu \mathrm{m}$ square microchannels using $300 \mathrm{~nm}$ diameter spherical particles was reported by Santiago et al. [26]. The flow field around the cylindrical obstruction was studied by employing a mercury arc lamp. The method was named micro-PIV $(\mu \mathrm{PIV})$. In other words, micron-resolution particle image velocimetry is a modification of PIV, where the whole volume of the flow is illuminated and the depth of velocity measurements is assessed from the depth of focus of an imaging microscope objective. Figure 2 shows a graphical representation of a $\mu \mathrm{DPIV}$ system.

In [28] authors reported the results of $\mu \mathrm{PIV}$ flow velocity measurements inside a $30 \times 300 \mu \mathrm{m}$ rectangular channel. They investigated pressure-driven flow using $200 \mathrm{~nm}$

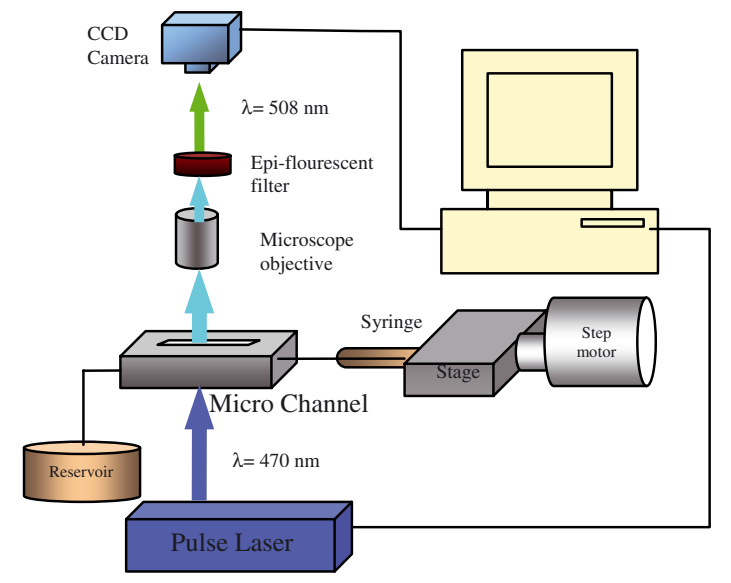

Fig. 2. Representation of the $\mu \mathrm{PIV}$ experimental setup.

particles, a 5 ns Nd:YAG laser and a cooled high resolution CCD camera. By employing the ensemble-averaged cross correlation method, velocities up to $10 \mathrm{~mm} / \mathrm{s}$ were recorded.

The background noise in mPIV, contributed by the out of focus particles, significantly depends on the particle concentration [31]. In the $\mu \mathrm{PIV}$ literature, there are discrepancies between definitions for the depth of field of an imaging microspore. Meinhart et al. in [32] defined the depth of focus in $\mu \mathrm{PIV}$ experiments by the equation:

$$
\delta z_{m}=\frac{3 n \lambda}{N A^{2}}+\frac{2.16 d_{p}}{\tan \theta}+d_{p} .
$$

This value indicates that the intensity contributed by particles out of this focus depth has a negligible influence on the correlation function. Errors arising from particle displacement assessments are another source of errors in $\mu \mathrm{PIV}$. According to [33], measurement error can be minimized if the particle image diameter projected onto the CCD sensor is approximately 3-4 pixels. In this condition, the position of the correlation peak can be determined with a precision of within one-tenth of the particle's image diameter. The diffraction-limited spot size $d_{s}$ of the infinity-corrected microscope optics that are used in $\mu \mathrm{PIV}$ applications is determined in [32] by the following equation:

$$
d_{s}=1.22 M \lambda \sqrt{\left(\frac{n}{N A}\right)^{2}}-1
$$

where, $M$ is the magnification, $N A$ is the numerical aperture, and $\mathrm{n}$ is the refractive index of the medium. Then, the effective diameter $d_{e}$ of the particle projected onto the CCD imaging plane, which is the convolution of the particle geometric image and the diffraction spot [33], can be calculated using:

$$
d_{e}=\sqrt{M^{2} d_{p}^{s}+d_{s}^{2}}
$$

where, $d_{p}$ is the particle diameter. Figure 3 shows a typical image of the $500 \mathrm{~nm}$ diameter micro-particle seeded flow.

During the last 10 years, the $\mu \mathrm{PIV}$ technique has been used in many research fields, for example, to study electroosmotic, pressure driven and mixed flow in a twin- $\mathrm{T}$ 


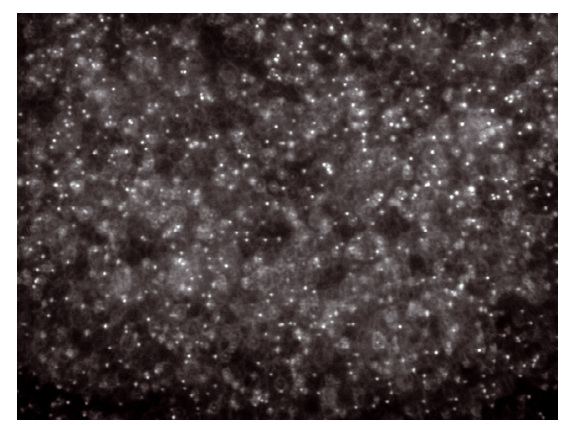

Fig. 3. $\mu \mathrm{PIV}$ imaging of fluorescent microparticle seeded flow.

microchannel intersection [34], thermal measurement dependent Brownian motion of microparticles [35], investigation of fluid slip mechanisms at wall [36] and in hydrophobic microchannels, internal circulation of aqueous plugs in two phases flow [37], in measurements of beads suspensions in water and human blood, using the red blood cells as a natural particle seeding [38]. A comprehensive review on the $\mu \mathrm{PIV}$ technique can be found in [39] and [40].

\section{Digital particle tracking velocimetry}

Generally, PIV means the accurate, quantitative measurement of fluid velocity vectors within a large number of points simultaneously. The other approach for the flow motion analysis is called Particle Tracking Velocimetry (PTV), where the displacement vector is determined by tracking the individual particles located at random points in the flow. On this account, the recent goal with digital particle tracking velocimetry (DPTV) systems is to determine by recording particle images digitally within the flow over multiple successive video frames and then conducting a completely computational analysis of the data. In comparison with traditional PIV techniques, one of the advantages of PTV is the ability to obtain more accuracy information about three-dimensional velocity vector by using volumetric illumination. Generally, a PTV algorithm consists of two successive steps: the detection an individual particle in frames and assessment of its displacement vector. One of the well-established PTV methods is the fourth-frame in-line tracking algorithm [41], where for the estimation of the best-match particle trajectory an iterative scheme involving extrapolation of the particle displacement and searching for the nearest neighbour is used. In [42] the binary-image cross-correlation method applied to digitally recorded particle images, the use of particle tracking algorithms, which perform a pointby-point matching of seed images from one frame to the next, allows construction of particle pathlines and instantaneous velocity field. A new algorithm for the PTV, where the performance of the particle tracking is much improved by employing the relaxation method, which for the individual-particle detection uses the dynamic thresholdbinarization method, was proposed in [43]. The stereoscopic 3D three -components PTV has been presented in [44]. It was shown that a single measurement can determine the in-plane velocity field throughout a $900 \times 720 \times$ $45 \mu \mathrm{m}$ volume. The out-of-plane resolution is limited only by the number of images taken and, ultimately, the size of the particles. In [45] a complete 3D PTV algorithm and some applications to indoor airflow velocity measurements are described. To recover longer trajectories, a temporal tracking algorithm was used. The results show that the algorithm is capable of tracking more than 1400 tracers in volumes up to $3 \mathrm{~m} \times 3 \mathrm{~m} \times 1.2 \mathrm{~m}$. In [46] the $3 \mathrm{D} \mathrm{PTV}$ was employed for investigation of turbulent pipe flow at high Reynolds numbers, where the study of the Lagrangian statistics of turbulent pipe flow has been carried out. The design parameters, selection criteria and solutions are discussed.

Generally, PTV is more accurate than the PIV, since the PTV algorithm tracks the three-dimensional coordinates of individual particles and the measured velocities are no longer automatically averaged onto the focal plane of the measurement. Thus, the PTV data represent a truly three-dimensional, three-components mapping of the flow field, whose resolution is independent of the microscope lens parameters. On the other hand, one of the main drawbacks of DPTV in comparison with PIV is that this technique can be applied to a low density distributed particle flow. On this account, there are a number of ideas about application of PTV algorithms to higher density particle images. For example, the "super-resolution" algorithm, introduced by Keane et al. [18], allows the extension of PTV algorithms to high particle density images. "Superresolution" algorithm employs the cross-correlation to obtain a PIV vector field and then uses this field to guide the search for particle matches in a subsequent PTV step [18]. Later, in [47] the hybrid PIV/PTV method is reported. The method consists of the standard cross-correlation PIV employed prior to individual-particle tracking in order to estimate in advance the local velocity to be measured by PTV. The other approach, combining PIV and CFD methods was presented in CFD [48], where the experimental particle-velocity data are compared iteratively with the theoretical expressions of the fluid flows. However, the problem with these methods is that they are not always effective, for instance, for detecting the small local structures.

\section{Conclusion}

A review of digital particle image velocimetry has been presented. The review examines the basics of particlebased visualization methods and DPIV, D $\mu$ PIV and DPTV techniques. The spatial and velocity dynamic range of PIV systems, in context of employing semiconductor imaging sensors, are explained and discussed.

Acknowledgements. We would like to thank Omer Altan for proofreading and correcting the English manuscript. 


\section{References}

1. http://nobelprize.org/

2. X. Huang, M.J. Gordon, R.N. Zare, Current-monitoring method for measuring the electroosmotic flow rate in capillary zone elec-trophoresis, Anal. Chem. 60, 1837 (1988)

3. S. Devasenathipathy, J.G. Santiago, Electro-kinetic flow diagnostics, Micro- and nano-scale diagnostic techniques, edited by K.S. Breuer (Springer, New York, Berlin, Heidelberg, 2004)

4. D. Sinton, Microscale flow visualization, Microfluid. Nanofluid. 1, 2 (2004)

5. A.K. Prasad, Particle image velocimetry, Curr. Sci. 79, 51 (2000)

6. A. Melling, Tracer particles and seeding for particle image velocimetry, Meas. Sci. Technol. 8, 1406 (1997)

7. A.K. Tieu, M.R. Mackenzie, E.B. Li, Measurements in microscopic flow with a solid-state LDA, Exp. Fluids 19, 293 (1995)

8. M. Minor, A.J. van der Linde, H.P. van Leeuwen, J. Lyklema, Dynamic aspects of electrophoresis and electroosmosis: a new fast method for measuring particle mobilities, J. Colloid Interface Sci. 189, 370 (1997)

9. D.A. Compton, J.K. Eaton, A high resolution laser Doppler anemometer for three-dimensional turbulent boundary layers, Exp. Fluids 22, 111 (1996)

10. R.J. Adrian, Twenty years of particle image velocimetry, Exp. Fluids 39, 159 (2005)

11. R.J. Adrian, C. Yao, Pulsed laser technique application to liquid and gaseous flows and the scattering power of seed materials, Appl. Opt. 24, 44 (1985)

12. E. Willert, M. Gharib, Digital particle image velocimetry, Exp. Fluids 10, 181 (1991)

13. R.J. Adrian, Particle imaging techniques for experimental fluid mechanics, Annu. Rev. Fluid Mech. 23, 261 (1991)

14. R.J. Adrian, Dynamic ranges of velocity and spatial resolution of particle image velocimetry, Meas. Sci. Technol. 8, 1393 (1997)

15. H. Huang, D. Dabiri, M. Gharib, On errors of digital particle image velocimetry, Meas. Sci. Technol. 8, 1427 (1997)

16. S.M. Hagsäter, C.H. Westergaard, H. Bruus, J.P. Kutter, Investigations on LED illumination for micro-PIV including a novel front-lit configurations, Exp. Fluids 44, 211 (2008)

17. O. Chételat, K.C. Kim, Miniature particle image velocimetry system with LED in-line illumination, Meas. Sci. Technol. 13, 1006 (2002)

18. R.D. Keane, R.J. Adrian, Y. Zhang, Super resolution particle image velocimetry, Meas. Sci. Technol. 6, 754 (1995)

19. R.D. Keane, R.J. Adrian, Theory of cross-correlation analysis of PIV images, Appl. Scientific Res. 49, 191 (1992)

20. F. Scarano, Iterative image deformation methods in PIV, Meas. Sci. Technol. 13, R1 (2002)

21. M.G. Olsen, R.J. Adrian, Brownian motion and correlation in particle image velocimetry, Opt. Laser Technol. 32, 621 (2000)
22. D.A. McQuarrie, Satistical mechanics (New York, Harper and Row, 1976)

23. James R. Janesick, Scientific Charge-Coupled Devices (SPIE Optical Engineering Press, WA, USA, 2001)

24. A. Hijazi, V. Madhavan, A novel ultra-igh speed camera for digital image processing applications, Meas. Sci. Technol. 19, 1 (2009)

25. D.R. Meldrum, M.R. Holl, Microscale bioanalytical systems, Science 297, 1197 (2002)

26. J.G. Santiago, S.T. Wereley, C.D. Meinhart, D.J. Beebe, R.J. Adrian, A particle image velocimetry system for microfluidics, Exp. Fluids 25, 316 (1998)

27. S. Devasenathipathy, J.G. Santiago, S.T. Wereley, C.D. Meinhart, K. Takehara, Particle imaging techniques for microfabricated fluidic systems, Exp. Fluids 34, 504 (2003)

28. C.D. Meinhart, S.T. Wereley, J.G. Santiago, PIV measurements of a microchannel flow, Exp. Fluids 27, 414 (1999)

29. K. Shinohara, Y. Sugii, A. Aota, A. Hibara, M. Tokeshi, T. Kitamori, K. Okamoto, High-speed micro-PIV measurements of transient flow in microfluidic devices, Meas. Sci. Technol. 15, 1965 (2004)

30. M.R. Bown, J.M. MacInnes, R.W.K. Allen, Micro-PIV measurementand simulation in complex microchannel geometries, Meas. Sci. Technol. 1, 619 (2005)

31. M.G. Olsen, C.J. Bourdon, Out-of-plane motion effects in microscopic particle image velocimetry, J. Fluids Eng. 125, $895(2003)$

32. C.D. Meinhart, S.T. Wereley, The theory of diffractionlimited resolution in microparticle image velocimetry, Meas. Sci. Technol. 14, 1047 (2003)

33. A.K. Prasad, R.J. Adrian, C.C. Landreth, P.W. Offutt, Effect of resolution on the speed and accuracy of particle image velocimetry interrogation, Exp. Fluids 13, 105 (1992)

34. J.M. MacInnes, X. Du, R.W. Allen, Prediction of electrokinetic and pressure ?ow in a microchannel T-junction, Phys. Fluids 15, 1992 (2003)

35. V. Hohreiter, S.T. Wereley, M.G. Olsen, J.N. Chung, Cross-correlation analysis for temperature measurement, Meas. Sci. Technol. 13, 1072 (2002)

36. D.C. Tretheway, C.D. Meinhart, A generating mechanism for apparent fluid slip in hydrophobic microchannels, Phys. Fluids 16, 1509 (2004)

37. C. King, E. Walsh, R. Grimes, PIV measurements of flow within plugs in a microchannel (2007), Vols. 3 and 4, pp. $463-472$

38. L. Bitsch, L.H. Olesen, C.H. Westergaard, H. Bruus, H. Klank, J.P. Kutter, Micro particle-image velocimetry of bead suspensions and blood flows (2005), Vol. 39, pp. 505511

39. R. Lindken, M. Rossi, S. Grosse, J. Westerweel, MicroParticle Image Velocimetry (microPIV): recent developments, applications, and guidelines, Lab. Chip. 9, 2551 (2009)

40. S.J. Lee, S. Kim, Advanced particle-based velocimetry techniques for microscale flows (2009), Vol. 6, pp. 577-588 
41. Y.A. Hassan, R.E. Canaan, Full-field bubbly flow velocity measurements using a multiframe particle tracking technique, Exp. Fluids 12, 49 (1991)

42. T. Uemura, F. Yamamoto, K. Ohmi, High speed algorithm of image analysis for real time measurement of two-dimensional velocity distribution Flow Visualization, edited by B. Khalighi et al. (ASME, FED-85, 1989), pp. $129-134$

43. K. Ohmi, H.Y. Li, Particle-tracking velocimetry with new algorithms, Meas. Sci. Technol. 11, 603 (2000)

44. M.R. Bown, J.M. MacInnes, R.W.K. Allen, W.B.J. Zimmerman, Three-dimensional, three-component velocity measurements using stereoscopic micro-PIV and PTV, Meas. Sci. Technol. 17, 2175 (2006)
45. P.H. Biwole, W. Yan, Y. Zhang, J. Roux, A complete 3D particle tracking algorithm and its applications to the indoor airflow study, Meas. Sci. Technol. 20, 115403 (2009)

46. R.J.E. Walpot, P.C.J.N. Rosielle, C.W.M. van der Geld, Design of a set-up for high-accuracy 3D PTV measurements in turbulent pipe flow, Meas. Sci. Technol. 17, 3015 (2006)

47. A. Kaga, K. Yamaguchi, A. Kondo, Y. Inoue, T. Yamaguchi, S. Kamoi, Flow field estimation using PIVdata and fluid dynamic equations, in Proc. PIV-Fukui '9'7 (1997), pp. 131-136

48. E.A. Cowen, S.G. Monismith, A hybrid digital particle tracking velocimetry technique, Exp. Fluids 22, 199 (1997) 